\title{
BMJ Open Maintenance motives for physical activity among older adults: a protocol for a systematic review and meta- analysis
}

\author{
Mary Katherine Huffman (D , ${ }^{1}$ Jason Brian Reed, ${ }^{2}$ Theresa Carpenter, ${ }^{1}$ \\ Steve Amireault (D) ${ }^{1}$
}

To cite: Huffman MK, Reed JB, Carpenter T, et al. Maintenance motives for physical activity among older adults: a protocol for a systematic review and meta-analysis. BMJ Open 2020;10:e032605. doi:10.1136/ bmjopen-2019-032605

- Prepublication history and additional material for this paper are available online. To view these files, please visit the journal online (http://dx.doi. org/10.1136/bmjopen-2019032605).

Received 26 June 2019 Revised 09 January 2020 Accepted 17 January 2020

Check for updates

(C) Author(s) (or their employer(s)) 2020. Re-use permitted under CC BY-NC. No commercial re-use. See rights and permissions. Published by BMJ.

${ }^{1}$ Health \& Kinesiology, Purdue University, West Lafayette, Indiana, USA

${ }^{2}$ Libraries and School of Information Studies, Purdue University, West Lafayette, Indiana, USA

Correspondence to

Dr Steve Amireault;

samireau@purdue.edu

\section{ABSTRACT}

Introduction Physical activity (PA) is an important aspect for health and well-being, yet many older adults do not maintain their PA long term. The identification of key factors that are associated with, and likely causally related to, older adults' PA maintenance is a crucial first step towards developing programmes that are effective at promoting long-term PA behaviour change. The purpose of this protocol is to outline a systematic review that will examine the relationship between four motives (ie, satisfaction, enjoyment, self-determination and identity) and older adults' PA maintenance.

Methods and analysis Studies that investigated PA maintenance with a sample mean age $\geq 55$ years will be included. Five electronic databases (PubMed, Cumulative Index to Nursing and Allied Health Literature, SPORTDiscus, PsycINF0 and ProQuest Dissertations and Theses) were searched on 6 April 2018 with no publication date limit (ie, from inception). One reviewer screened $100 \%$ of titles and abstracts $(\mathrm{k}=21470)$ while a random subsample $(20 \%)$ was screened independently by two reviewers. An update of the search was run on 1 October 2019. All studies for which the full text was retrieved will be independently screened by two reviewers. Data pertaining to study sample, design, motives, PA (eg, measurement validity evidence, study definition of maintenance) and essential bias domains (eg, bias due to missing data) will be extracted. Study-level effect sizes will be calculated, and if the number of studies is $\geq 5$, a random-effects meta-analysis will be performed using inverse-variance methods; a narrative synthesis will be performed otherwise.

Ethics and dissemination The university's Human Research Protection Program determined that the proposed study qualifies as exempt from the Institutional Review Board review under Exemption Category 4 (PROPEL \#: 80047007). Results will be published in a peerreview journal, and the findings will help inform future interventions with older adults.

PROSPERO registration number CRD42018088161.

The benefits of engaging in physical activity (PA) are well known and can be experienced across the lifespan. For example, PA has been linked to improved quality of life, ${ }^{1}$
Strengths and limitations of this study

- The proposed review includes a comprehensive literature search strategy that specifically focuses on proposed theory-based maintenance motives.

- Planned subgroup analyses will provide more specific insight regarding for whom the motives are more or less influential.

- A low number of studies and significant heterogeneity between studies might preclude performing meta-analyses of the results.

- Publication bias may be difficult to appraise if it is not possible to compute study-level effect sizes and/ or if there is not a sufficient number of studies.

prevention of cardiovascular disease and osteoporosis, management of chronic conditions (eg, arthritis $)^{2}$ and reduced age-related physical and cognitive decline ${ }^{3}$ for older adults. Although PA is a vital aspect for maintenance of human health and well-being, maintaining regular engagement in PA remains challenging for older adults. ${ }^{45}$ For instance, there is mixed evidence supporting the effectiveness of behavioural interventions regarding the maintenance of PA beyond programme termination, ${ }^{67}$ and declines in PA levels are common for older adults after participation in such programmes. ${ }^{89}$ This represents an important public health challenge because stopping or reducing PA can result in a significant reversal of initial health improvements. ${ }^{2} 1011$ Collectively, this information highlights the need to devote special attention to the design of interventions that are more conducive of maintaining PA over time.

One of the first essential steps towards helping older adults maintain their PA behaviour is to identify the most influential factors associated with, and likely causally related to, the maintenance of that behaviour. ${ }^{12}$ The identification of such factors 
Table 1 Maintenance motives for behaviour change maintenance

\begin{tabular}{lll}
\hline Maintenance motive & Definition & Theoretical basis for maintenance \\
\hline $\begin{array}{l}\text { Satisfaction with outcomes and } \\
\text { behavioural enjoyment }\end{array}$ & $\begin{array}{l}\text { An individual's positive self-assessment } \\
\text { of the relative costs and benefits } \\
\text { afforded by the behaviour and the } \\
\text { behavioural experiences. }\end{array}$ & $\begin{array}{l}\text { Sactory physical activity outcomes and } \\
\text { experiences enhance the tendency to repeat the } \\
\text { action by reinforcing the decision to engage in the } \\
\text { behaviour. }\end{array}$ \\
$\begin{array}{lll}\text { An individual's free choice to engage in } \\
\text { a behaviour. }\end{array}$ & $\begin{array}{l}\text { Physical activity is more likely to be maintained } \\
\text { when it is personally relevant, valued and } \\
\text { autonomously chosen. }\end{array}$ \\
Identity & $\begin{array}{l}\text { An individual's sense of self, including } \\
\text { values, beliefs and needs. }\end{array}$ & $\begin{array}{l}\text { The degree to which one's identity is congruent } \\
\text { with physical activity fosters internalisation and } \\
\text { behavioural regulation. }\end{array}$ \\
\hline
\end{tabular}

Adapted from Kwasnicka et al. ${ }^{13}$

offers foundational insights into what needs to be modified in any future behavioural interventions and thus provides judicious guidance for selecting programme components (eg, behaviour change techniques or strategies) that maximally promote the targeted behaviour. Therefore, a knowledge synthesis of theory-based motives related to PA maintenance in older adults would represent one valuable tool that programme developers and researchers could use to ultimately design evidence-based behavioural programmes that are more effective for that population.

\section{THEORETICAL RATIONALE}

According to a recent framework synthesising theoretical explanations for the maintenance of behaviour change, ${ }^{13}$ maintenance can be conceptualised through five overarching themes: maintenance motives, self-regulation, resources, habit and environmental and social influences. Maintenance motives are hypothesised to be different from those motives that underlie the initial adoption of a behaviour. The maintenance motive theme consists of three concepts: satisfaction with behavioural outcomes and enjoyment of the behaviour, self-determination and identity (see table 1). Moreover, maintenance motives are the primary drivers for behaviour; they influence priorities, decisions regarding the distribution of relevant resources and decisions regarding self-regulation. ${ }^{13}$ Although this framework proposes that these motives are pertinent for all health behaviours and all populations, it is currently unknown if this review-level postulation can generalise to PA behaviour or to the older adult population. Therefore, the main purpose of this proposed review is to examine if, and to what extent, the theorised maintenance motives are related to the maintenance of PA for older adults.

In the PA literature, the term 'maintenance' can refer to different behavioural contexts. ${ }^{14}$ First, maintenance of an intervention-induced change in PA refers to situations in which inactive individuals who increased their PA in response to participating in a behavioural intervention are still regularly active for a given period of time beyond programme termination. Second, maintenance of self-initiated PA can refer to situations in which inactive individuals increased their PA on their own (ie, without participating in a PA programme) and are still regularly active for a given period of time, as well as situations in which individuals have always been physically active. ${ }^{14}$ It is worth noting that people who try to maintain PA beyond programme termination may form a more homogeneous group in terms of their experiences with PA behaviour compared with those who try to maintain self-initiated PA. ${ }^{15}$ Therefore, these two maintenance contexts will be considered in subgroup analyses.

\section{Previous reviews of correlates of PA maintenance}

Although many reviews exist examining correlates of PA behaviour (eg, see Bauman et al ${ }^{16}$ for a review of systematic reviews about the correlates of $\mathrm{PA}$ in children and adults), few have examined the correlates of PA maintenance specifically. Rhodes and Quinlan ${ }^{17}$ and Amireault et $a l^{15}$ systematically reviewed studies of adults ages 18-64 and concluded that intention is a predictor of PA change and maintenance. Regarding older adults, a narrative review by Rhodes and Quinlan ${ }^{18}$ concluded that exercise history, self-efficacy and social support were related to regular exercise; however, most included studies were cross-sectional. A later systematic review by van Stralen et $a l^{19}$ reported that outcome expectations and action planning were associated with PA initiation, while coping planning and outcome realisation were associated with maintenance across studies for older adults. Additionally, these previous reviews investigated 'correlates', 'predictors' or 'determinants', resulting in a broad scope of factors related to PA maintenance. To summarise, few reviews have assessed factors related to PA maintenance in the older adult population, and no review has yet to focus on the aforementioned theorised maintenance motives specifically.

The proposed review will expand on these previous reviews in several ways. First, it will explicitly examine the factors of identity, self-determination, satisfaction and enjoyment. Second, this review is designed to assess study-level effect sizes and perform meta-analyses rather 
than synthesise results using vote-counting procedures, as previous reviews of older adults have done. ${ }^{18-20}$ Third, the proposed review will target older adults ages 55 and older and conduct planned subgroup analyses to impart knowledge regarding for which subgroups of the targeted population these maintenance motives are more or less influential. We will perform subgroup analyses based on sample health status, as Amireault et $a l^{15}$ found support for health status (apparently healthy adults vs adults with chronic disease or disability) as a potential moderating variable in their systematic review. Furthermore, Rhodes and Quinlan ${ }^{17}$ have suggested that adults over the age of 64 may require separate reviews, and research has indicated that women and men may differ in some sources of motivation (eg, general social support may be more influential for women's PA) ${ }^{21}$; therefore, we plan to perform subgroup analyses to determine whether the relationships between the maintenance motives and PA vary as a function of sample mean age and percentage of women in the studied sample. Finally, we will perform a subgroup analysis based on study maintenance context (ie, maintenance of self-initiated PA vs maintenance of PA beyond programme termination).

\section{Objectives}

The objective of the proposed systematic review is to evaluate the extent to which the maintenance motives are related to physical activity maintenance among older adults aged 55 and older. This review will address the following questions:

1. What maintenance motives are related to PA maintenance for older adults?

2. Which maintenance motives are most strongly related to PA maintenance for older adults?

3. Does the predictive capacity of the maintenance motives vary according to the following sample characteristics: (1) age, (2) gender distribution, (3) health status (eg, cancer, diabetes) and/or (4) maintenance context?

\section{METHODS AND ANALYSIS}

This protocol follows the Preferred Reporting Items for Systematic Review and Meta-Analysis Protocols (PRISMA-P) 2015 guidelines for the description and reporting of systematic review protocols. ${ }^{22}$ Any amendments to the protocol will be tracked and dated in PROSPERO. A copy of the PRISMA-P checklist is included in online supplementary appendix 1 .

\section{Study eligibility criteria}

Studies that investigated PA maintenance in an older adult population with a mean age of 55 or older were included. We acknowledge that defining the older adult population as 55 years or older is somewhat arbitrary; however, previous research has used this delineation, arguing that the age group of 55-64 serves as a point of reference marking the age-related decline in health. ${ }^{23}$
The study designs that were included are longitudinal, experimental/randomised controlled trials, quasiexperimental and one-group pretest-post-test studies which assessed PA at least twice (including a follow-up assessment for intervention studies) and at least one maintenance motive. No restrictions were placed on the length of the study, follow-up or type of intervention or control group. These types of study designs were purposefully included to assess the relationships between the motives and PA behaviour within the two maintenance contexts. ${ }^{14}$ Cross-sectional and qualitative studies, books and book chapters were excluded. No constraints were placed on the publishing year, country or the language of the studies included.

Furthermore, although Kwasnicka et $a l^{13}$ consider enjoyment and satisfaction to be largely the same maintenance motive, from herein, we consider the two as different motives and thus will assess each separately. Although there may be overlap between the two, especially in the PA domain, ${ }^{24}$ it is possible that the studies we retrieve likewise consider them separately (eg, enjoyment of PA, satisfaction with PA experiences and outcomes).

It is also worth mentioning that there is a lack of consensus throughout the literature regarding the conceptualisation of maintenance and even some confusion concerning the difference between adherence and uptake. ${ }^{15} 1925$ However, we assert that the maintenance of PA is not an unwavering continuation of behaviour; it is a process that may include multiple episodes of sustained engagement in PA that can be discontinued for a short (lapse) or a longer (relapse) period of time and then resumed after a setback (recovery). ${ }^{142627}$ Thus, to capture our notion of maintenance while acknowledging others' understanding and past use of the concept, study-specific assessments of PA maintenance were not restricted to a particular study design, follow-up duration or analysis method (eg, difference scores, residual change scores, dichotomous change scores-relapse vs maintenance, within-person changes) in this review. The requirement is that the studies included must have assessed PA at least twice, thereby providing an indication of PA trajectories over time. Thus, we will include studies that are controlling, either statistically or by design, for past PA behaviour-a potential confounding variable. ${ }^{13}$

\section{Information sources and search strategies}

An electronic databases (coverage period) search strategy was developed by the review team, including a database expert and health sciences information specialist, for PubMed (1946-2019), PsycINFO (EBSCO interface; 1887-2019), SPORTDiscus (EBSCO interface; 1800-2019), Cumulative Index to Nursing and Allied Health Literature (CINAHL) (EBSCO interface; 1976-2019) and ProQuest Dissertations and Theses (1637-2019; full-text coverage: 1997-2019). For all databases, search terms for the maintenance motives, 
PA and maintenance were used. Additionally, databasespecific Index or Medical Subject Headings terms were used when available. The free-text search terms remained constant across all databases, searching across title, abstract and when available, keyword fields. The database-specific terms were updated for each database, where available, but were identified using the same concepts across all the databases. No date or language filters were used in any of the databases. Filters for resource type (academic journals or dissertations) were used in two of the EBSCO databases (SPORTDiscus and PsycINFO) because of the indexing of periodicals in EBSCO. This option was not used for CINAHL because the filter was experiencing technical difficulties when the searches were run. Full details of an example electronic search for PubMed are presented in online supplementary appendix 2. A hand search of the reference lists for all eligible full-text articles retrieved and relevant literature reviews ${ }^{15}{ }^{17-20}$ will also be performed to identify additional citations to assess for eligibility. All retrieved literature citation records, after removing duplicates, were uploaded to Rayyan QCRI Web application. ${ }^{28}$ Rayyan QCRI is a Web application that aids in the housing and screening of abstracts and titles. The results of the search will be reported in a PRISMA flowchart. $^{29}$

\section{Study selection}

The initial search was run on 6 April 2018. Two reviewers (MKH and TC) independently screened titles and abstracts and excluded clearly irrelevant studies. Specifically, one reviewer $(\mathrm{MKH})$ independently screened $100 \%$ of the titles and abstracts $(\mathrm{k}=21470)$. The second reviewer (TC) independently screened $20 \%$ of the titles and abstracts. Reviewers were instructed to be overinclusive at this stage of the screening process. If there was insufficient information to certainly conclude that a given citation should be excluded, the citation was retained and included in the full-text screening stage. The decisions were compared, and discrepancies among the two reviewers were resolved by discussion. At the end of this process, the reviewers disagreed on only one study (ie, $0.023 \%$ of the titles and abstracts screened by the two reviewers). The review team concluded that there would likely be no added benefits-but additional time and cost constraints-of having a second reviewer independently screen the remaining $80 \%$ of titles and abstracts. Therefore, only one reviewer independently screened all 21470 titles and abstracts. The full text of the articles decided for inclusion at the title and abstract screening stage were retrieved, and two reviewers (MKH and SA) will independently assess the eligibility of each article; the results will be compared, and discrepancies between reviewers will be resolved by discussion. When no consensus can be reached, a third reviewer (JBR) will help resolve the discrepancy. Note that the search was updated on 1 October 2019 and resulted in two additional relevant articles to be included in the review.

\section{Data items and data extraction}

Prior to data extraction, two reviewers (MKH and SA) will independently pilot a purpose-built checklist with three randomly selected studies. The checklist will be designed to collect information on the characteristics of the study (eg, sample size, maintenance context, year of publication, country in which the study was conducted), the maintenance motive measured (eg, measurement instrument used, measurement validity evidence), the sample (eg, mean age, percentage of women, ethnicity, level of education, retirement status, health status, marital status) and the PA assessed (main outcome; for example, study definition of PA maintenance, measurement instrument used, measurement validity evidence); specifically, reviewers will extract relevant information regarding all types of PA, which may include leisure time PA (eg, walking), sport participation and/or exercise. All PA units (frequency, duration, volume) will be considered, as well as assessments via objective measures (eg, accelerometers) and self-report. In addition, data required for study-level effect size calculation (eg, mean, correlation coefficient, OR, SD, analysed sample size, F-test, $\chi^{2}$ and t-test values and $p$ value) will be extracted. Following the pilot testing, the same reviewers will independently extract data from all included studies, compare results and resolve any discrepancies through discussion. When no consensus can be reached, a third reviewer (JBR) will help resolve the discrepancy. Authors of primary studies will be contacted (maximum of three email attempts over a maximum of 5 weeks) to obtain missing information. Relevant information pertaining to the risk of bias in primary studies ${ }^{30}$ will be retrieved. More details regarding risk of bias are presented in the following section and in online supplementary appendix 3.

\section{Risk of bias appraisal}

The assessment of the risk of bias in primary studies will be conducted using an adapted version of the Risk of Bias In Non-randomised Studies-of Interventions (ROBINS- $\mathrm{I}^{31}$ ). Accordingly, the following relevant bias domains will be assessed: (1) bias due to confounding; (2) bias in selection of participants into the study; (3) bias in measurement of the outcome (ie, physical activity); (4) bias in the measurement of the exposure (ie, maintenance motives); (5) bias due to missing outcome data and (6) bias in selection of the reported results. For each bias domain, a set of signalling questions will provide guidance for eliciting relevant information about each bias domain. Because our systematic review is considering observational studies, including the postintervention features of intervention studies (ie, follow-up from programme termination), additional relevant signalling questions were drawn from a checklist for the assessment of the methodological quality of non-randomised studies of healthcare interventions. ${ }^{32}$ The response options for all signalling questions are: yes; probably yes; probably no; no; no information. See online supplementary appendix 3 for the risk of bias tool that will be used. Free text boxes 
will also be available to the reviewers to provide justification responses in support of their answers to signalling questions and risk of bias judgements for each domain. Three qualitative ratings reflecting the risk of bias judgement will be assigned to each bias domain ${ }^{33}$ : low risk of bias (plausible bias unlikely to seriously alter the results), some concerns (plausible bias that raises doubt about the results) and high risk of bias (plausible bias that seriously weakens confidence in the results). We will consider bias domains and their corresponding rating independently without making an overall risk of bias judgement for each primary study. Data will be compared between two reviewers (MKH and SA), and discrepancies will be resolved by discussion until consensus is reached. When no consensus can be reached, a third reviewer (JBR) will help resolve the discrepancy.

\section{Data analysis}

Primary study characteristics (eg, sample size, sample mean age, percentage of female participants, percentage of Caucasian/white participants, study publication year) will be reported for descriptive purposes in a summary table. The study-level effect size, such as odds ratio (OR) for dichotomous outcomes and standardised mean differences (SMD) or Pearson correlation coefficient $(r)$ for continuous outcomes will be calculated and reported. The OR and SMD effect sizes will be converted to Pearson correlation coefficients to facilitate interpretation and to allow between-study comparison.

\section{Quantitative synthesis}

If the number of included primary studies for a given maintenance motive is $\geq 5$, a random-effects meta-analysis will be performed using Comprehensive Meta-Analysis software, V.3 (Biostat; Englewood, New Jersey, USA, 2014). The inverse-variance method will be used for all meta-analyses. ${ }^{34}$ Subgroup analyses will be performed according to the following variables: population (eg, samples with or without health conditions), participant characteristics (sample mean age, percentage of women in the sample) and maintenance context (ie, maintenance of self-initiated PA, maintenance of PA beyond programme termination). If the ratio of number of studies for each key covariate $\geq 10$, meta-regression analysis will be performed instead of subgroup analyses. ${ }^{35}$ Further variation in effect sizes will be examined with respect to risk of bias in primary studies.

\section{Heterogeneity inspection}

Variation in the magnitude and direction of primary effect sizes will be assessed using both qualitative and quantitative criteria $^{36}$ by: describing the variation in study-level effect sizes; verifying the amount of overlap (none, minimal or substantial) in 95\% CIs; performing the Cochran $\mathrm{Q} \chi^{2}$ test, which tests the hypothesis that all studies share a common effect size $(p<0.10)$; and reporting the percentage of total variation in estimated effects that is due to among-study variation rather than chance $\left(\mathrm{I}^{2}\right)$. An $\mathrm{I}^{2}$ value of $25 \%$ is considered to reflect low heterogeneity, $50 \%$ moderate heterogeneity and $75 \%$ high heterogeneity. ${ }^{37}$ If heterogeneity is substantial, the summary effect sizes will not be reported; a narrative synthesis will be done instead.

\section{Narrative synthesis}

If there is a substantial difference in study sample, or heterogeneity in study-level effect size, a three-step process will be used to synthesise the body of evidence for each maintenance motive. First, study-level effect sizes for each of the studied maintenance motives will be organised in a table based on the frequency at which a given maintenance motive was examined and study sample size. Second, sample characteristics (eg, mean age, percentage of female participants, sample health status, maintenance context) will also be organised and displayed in a table. Third, visual inspection of the data displays using Boxand-Whisker plots for all maintenance motive study-level effect sizes will be used to informally examine whether the distribution of effects differs as a function of sample mean age, percentage of female participants, sample health status, risk of bias assessment ratings, maintenance context and sample size. Within-study moderation or subgroup analysis findings with respect to these characteristics (gender, age, health status and maintenance context) will also be noted.

\section{Publication bias}

Assessment of publication bias will rely on the following assumptions. First, studies, irrespective of their sample size, reporting statistically significant results $(\mathrm{p}<0.05)$ are more likely to be published compared with studies reporting non-statistically significant results. Second, small sample size studies-especially those reporting nonstatistically significant results $(\mathrm{p} \geq 0.05)$-are at the greatest risk for being unpublished. Under such circumstances, small sample size studies reporting the strongest effects are therefore more likely to report statistically significant results (and get published). Conversely, smaller sample size studies reporting trivial, small and even moderate effect sizes are more likely to remain unpublished. Taken together, the risk of publication bias in systematic reviews will likely increase as the number of small sample size studies included in the review increases. ${ }^{38-40}$ Therefore, overall likelihood of publication bias will be appraised when there are at least 10 study-level effect sizes for the same maintenance motive. ${ }^{41}$ First, we will conduct a cumulative meta-analysis, where primary studies will be plotted from the most precise to least precise-larger studies will appear towards the top and smaller studies will appear towards the bottom of the forest plot. Publication bias will be suspected if the effects shift (either to the left or right) as we move towards the bottom of the forest plot. ${ }^{42}$ Second, discrepancy in findings between published studies and dissertation and thesis documents (unpublished studies) will be assessed. Finally, we will visually inspect the distribution of the funnel plot and use the Egger's regression test. ${ }^{38}$ 


\section{Quality of evidence}

The quality of evidence for each maintenance motive will be assessed across the domains of risk of bias, consistency of the results, precision and magnitude of the effect (if a meta-analysis is performed) and publication bias, using the Grading of Recommendations Assessment, Development and Evaluation approach. ${ }^{43}$

\section{Patient and public involvement}

No patient involved.

\section{ETHICS AND DISSEMINATION PLAN AND IMPLICATIONS}

The results of this review will be published in a peerreviewed journal and presented at relevant scientific conferences. In addition, results will be communicated to members of the target population (ie, adults ages 55 or older) at relevant community talks. Importantly, the conclusions of this review will help inform future interventions regarding how to maintain PA for older adults.

Contributors All listed authors have contributed and will continue to contribute meaningfully to the protocol and proposed review. MKH and SA conceived the proposed review. JBR, MKH and SA developed the search strategy, and JBR ran the pilot search as well as the final search. MKH and TC are the two title and abstract reviewers, and MKH and SA are the two full-text reviewers. JBR will be the third reviewer that will help resolve any discrepancy. MKH submitted the protocol to PROSPERO and is responsible for updating the registered protocol as needed. All authors read the final protocol manuscript and revised it for content; all also approved the final version.

Funding The authors have not declared a specific grant for this research from any funding agency in the public, commercial or not-for-profit sectors.

Competing interests None declared.

Patient consent for publication Not required.

Ethics approval The university's Human Research Protection Program determined that the proposed study qualifies as exempt from Institutional Review Board review, under federal human subjects research regulations Exemption Category 4 (PROPEL \#: 80047007).

Provenance and peer review Not commissioned; externally peer reviewed.

Open access This is an open access article distributed in accordance with the Creative Commons Attribution Non Commercial (CC BY-NC 4.0) license, which permits others to distribute, remix, adapt, build upon this work non-commercially, and license their derivative works on different terms, provided the original work is properly cited, appropriate credit is given, any changes made indicated, and the use is non-commercial. See: http://creativecommons.org/licenses/by-nc/4.0/.

\section{ORCID iDs}

Mary Katherine Huffman http://orcid.org/0000-0003-3396-5565

Steve Amireault http://orcid.org/0000-0003-3372-2555

\section{REFERENCES}

1 Elavsky S, McAuley E, Motl RW, et al. Physical activity enhances long-term quality of life in older adults: efficacy, Esteem, and affective influences. Ann Behav Med 2005;30:138-45.

2 Chodzko-Zajko WJ, Proctor DN, Fiatarone Singh MA, et al. Exercise and physical activity for older adults. Med Sci Sports Exerc 2009;41:1510-30.

3 Muscari A, Giannoni C, Pierpaoli L, et al. Chronic endurance exercise training prevents aging-related cognitive decline in healthy older adults: a randomized controlled trial. Int $J$ Geriatr Psychiatry 2010;25:1055-64.

4 Brawley LR, Rejeski WJ, King AC. Promoting physical activity for older adults: the challenges for changing behavior. Am J Prev Med 2003;25:172-83.
5 Hughes SL, Leith KH, Marquez DX, et al. Physical activity and older adults: expert consensus for a new research agenda. Gerontologist 2011;51:822-32.

6 Conn VS, Minor MA, Burks KJ, et al. Integrative review of physical activity intervention research with aging adults. J Am Geriatr Soc 2003;51:1159-68.

7 van der Bij AK, Laurant MGH, Wensing M. Effectiveness of physical activity interventions for older adults: a review. Am J Prev Med 2002;22:120-33.

8 Hertogh EM, Vergouwe $\mathrm{Y}$, Schuit AJ, et al. Behavioral changes after a 1-yr exercise program and predictors of maintenance. Med Sci Sports Exerc 2010;42:886-92.

9 McAuley E, Morris KS, Motl RW, et al. Long-Term follow-up of physical activity behavior in older adults. Health Psychol 2007:26:375-80.

10 Mujika I, Padilla S. Detraining: loss of training-induced physiological and performance adaptations. Part II: long term insufficient training stimulus. Sports Med 2000;30:145-54.

11 Mujika I, Padilla S. Cardiorespiratory and metabolic characteristics of detraining in humans. Med Sci Sports Exerc 2001;33:413-21.

12 Sheeran P, Klein WMP, Rothman AJ. Health behavior change: moving from observation to intervention. Annu Rev Psychol 2017;68:573-600.

13 Kwasnicka D, Dombrowski SU, White M, et al. Theoretical explanations for maintenance of behaviour change: a systematic review of behaviour theories. Health Psychol Rev 2016;10:277-96.

14 Marcus BH, Dubbert PM, Forsyth LH, Stone EJ, et al. Physical activity behavior change: issues in adoption and maintenance. Health Psychol 2000;19:32-41.

15 Amireault S, Godin G, Vézina-Im L-A. Determinants of physical activity maintenance: a systematic review and meta-analyses. Health Psychol Rev 2013;7:55-91.

16 Bauman AE, Reis RS, Sallis JF, et al. Correlates of physical activity: why are some people physically active and others not? Lancet 2012;380:258-71.

17 Rhodes RE, Quinlan A. Predictors of physical activity change among adults using observational designs. Sports Med 2015;45:423-41.

18 Rhodes RE, Martin AD, Taunton JE, et al. Factors associated with exercise adherence among older adults. An individual perspective. Sports Med 1999;28:397-411.

19 van Stralen MM, De Vries $\mathrm{H}$, Mudde AN, et al. Determinants of initiation and maintenance of physical activity among older adults: a literature review. Health Psychol Rev 2009;3:147-207.

20 Koeneman MA, Verheijden MW, Chinapaw MJM, et al. Determinants of physical activity and exercise in healthy older adults: a systematic review. Int J Behav Nutr Phys Act 2011;8:142.

21 Lindsay Smith G, Banting L, Eime R, et al. The association between social support and physical activity in older adults: a systematic review. Int J Behav Nutr Phys Act 2017;14:56.

22 Moher D, Shamseer L, Clarke M, et al. Preferred reporting items for systematic review and meta-analysis protocols (PRISMA-P) 2015 statement. Syst Rev 2015;4:1.

23 Schoenborn CA, Heyman KM. Health characteristics of adults aged 55 years and over: United States, 2004-2007. Natl Health Stat Report 2009;16:1-31.

24 Baldwin AS, Sala M, Williams DM, et al. Percieved satisfaction with health behavior change. In: Affective determinants of health behavior. New York: NY: Oxford University Press, 2018: 69-89.

25 Hawley-Hague H, Horne M, Skelton DA, et al. Review of how we should define (and measure) adherence in studies examining older adults' participation in exercise classes. BMJ Open 2016:6:e011560.

26 Kahlert D. Maintenance of physical activity: do we know what we are talking about? Prev Med Rep 2015;2:178-80.

27 Sallis JF, Hovell MF. Determinants of exercise behavior. Exerc Sport Sci Rev 1990;18:307???330-30.

28 Ouzzani M, Hammady H, Fedorowicz Z, et al. Rayyan-a web and mobile APP for systematic reviews. Syst Rev 2016;5:210.

29 Moher D, Liberati A, Tetzlaff J, et al. Preferred reporting items for systematic reviews and meta-analyses: the PRISMA statement. PLoS Med 2009;6:e1000097.

30 Guyatt GH, Oxman AD, Vist G, et al. GRADE guidelines: 4. Rating the quality of evidence--study limitations (risk of bias). J Clin Epidemiol 2011;64:407-15.

31 Sterne JA, Hernán MA, Reeves BC, et al. ROBINS-I: a tool for assessing risk of bias in non-randomised studies of interventions. BMJ 2016;355:i4919-7.

32 Downs SH, Black N. The feasibility of creating a checklist for the assessment of the methodological quality both of randomised and non-randomised studies of health care interventions. J Epidemiol Community Health 1998;52:377-84. 
33 Higgins JPT, Altman DG, Sterne JAC. Cochrane Handbook for systematic reviews of intervention, 2011. Available: https://training cochrane.org/handbook [Accessed 24 Jun 2019].

34 Higgins JPT, Thompson SG, Spiegelhalter DJ. A re-evaluation of random-effects meta-analysis. J R Stat Soc Ser A Stat Soc 2009;172:137-59.

35 Borenstein M, Hedges LV, Higgins JPT, et al. Introduction to metaanalysis. John Wiley \& Sons, 2009: 187-203.

36 Guyatt GH, Oxman AD, Kunz R, et al. GRADE guidelines: 7. Rating the quality of evidence--inconsistency. J Clin Epidemiol 2011;64:1294-302.

37 Higgins JPT, Thompson SG, Deeks JJ, et al. Measuring inconsistency in meta-analyses. BMJ 2003;327:557-60.
38 Egger M, Davey Smith G, Schneider M, et al. Bias in meta-analysis detected by a simple, graphical test. BMJ 1997;315:629-34.

39 Guyatt GH, Oxman AD, Montori V, et al. GRADE guidelines: 5 . Rating the quality of evidence--publication bias. J Clin Epidemiol 2011;64:1277-82.

40 loannidis JPA. Why most published research findings are false. PLOS Med 2005:2:e124.

41 Sutton AJ, Duval SJ, Tweedie RL, et al. Empirical assessment of effect of publication bias on meta-analyses. BMJ 2000;320:1574-7.

42 Borenstein M, Hedges LV, Higgins JPT, et al. Introduction to metaanalysis. John Wiley \& Sons, 2009: 277-92.

43 Balshem H, Helfand M, Schünemann HJ, et al. Grade quidelines: 3 . rating the quality of evidence. J Clin Epidemiol 2011;64:401-6. 